\title{
Análise de tendência da sinistralidade e impacto na diminuição do número de operadoras de saúde suplementar no Brasil
}

\author{
Trend analysis of the claim rate and the impact on the reduction \\ of the number of private healthcare providers in Brazil
}

Ângelo Augusto da Silva Araújo ${ }^{1}$

José Rodrigo Santos Silva ${ }^{2}$

${ }^{1}$ Universidad de Ciencias Empresariales y Sociales. Paraguay 1401, C1061ABA CABA. Buenos Aires Argentina. angeloaugusto@ hotmail.com

${ }^{2}$ Departamento de

Estatística e Ciências

Atuárias, Universidade

Federal de Sergipe. São

Cristóvão SE Brasil.

\begin{abstract}
Alarming data on the part of health care providers on the increase of the claim rate and its potential risk has emerged. It is a descriptive study, with the objective of understanding the changes in the healthcare provider sector in recent years, using the temporal analysis of historical series related to the sector. The variables selected for this study were the claim rate, the coverage rate, and the number of private healthcare providers in activity, observed from 2003 to 2014. The method used for evaluation of the temporal trend was Linear Regression. The claim rate and the coverage rate show an upward trend in the period, while the number of operators in Brazil showed a decreasing trend during the same period. These results show that even with the increase in demand, there was a decrease in the number of operators active in the country. The claim rate is one of the possible causes observed this inverse relationship because the increased offers risks of survival and the opening of new operators. Moreover, the decrease in the number of providers, is leading the country to an oligopolistic industry with an increasing demand in the number of beneficiaries. This decrease is also associated with regulatory processes, which regulates the sector's relationship with the beneficiary.

Key words Claim rate, Healthcare, Trend analyses
\end{abstract}

Resumo Dados alarmantes vêm surgindo por parte das operadoras de saúde sobre o aumento da sinistralidade e seu potencial risco. Estudo descritivo, com o objetivo de compreender as mudanças ocorridas no setor de saúde suplementar nos últimos anos, através da análise temporal de séries históricas relacionadas ao setor. As variáveis escolhidas para este trabalho foram a sinistralidade, a taxa de cobertura e o quantitativo de operadoras em atividade, observadas de 2003 a 2014. O método utilizado para a avaliação da tendência temporal foi a Regressão Linear. A sinistralidade e a taxa de cobertura apresentaram uma tendência de crescimento no período, enquanto a quantidade de operadoras no Brasil apresentou uma tendência de decrescimento no mesmo período. Esses resultados apontam que, mesmo com o aumento da demanda, houve uma diminuição do número de operadoras em atividade no país. A sinistralidade é uma das possíveis causas de observarmos essa relação inversa, pois o seu aumento oferece riscos à sobrevida e à abertura de novas operadoras. Ademais, a diminuição do número de operadoras está conduzindo o país a uma oligopolização do setor com uma demanda crescente do número de beneficiários. Essa diminuição pode estar também associada aos processos regulatórios que normatizam a relação do setor com o beneficiário.

Palavras-chave Sinistralidade, Plano de saúde, Análise de tendência 


\section{Introdução}

Na década de 1970, o grande desenvolvimento tecnológico e medicamentoso associado à política flexneriana e ao interesse mercadológico na área de saúde (devido à grande demanda e pouca oferta) fez com que o governo brasileiro incentivasse o crescimento significativo do setor privado de saúde, financiando sua expansão e renovação, e, de certo modo, garantindo a compra dos serviços, fazendo com que o setor de saúde crescesse fortemente nesse período ${ }^{1}$. Segundo Ligia Bahia ${ }^{2}$, o setor de saúde foi levado a uma universalização perversa, americanizada e excludente na política social brasileira. Escrivão Júnior e Koyama ${ }^{1}$ descrevem que na década de 1990 houve grande incentivo do governo ao crescimento dos prestadores privados de assistência médica, os quais obtinham financiamentos para a expansão e o incremento dos serviços, além da garantia de demanda, sendo o Estado um importante comprador dos serviços. Em tal ambiente, em que a sobrevivência e a viabilidade empresarial estavam, de certa forma, garantidas por mecanismos como o acima exposto, não eram priorizadas, nas empresas do setor suplementar, medidas para melhorar os processos e o atendimento, sendo pequena a ênfase no conjunto: qualidade, eficácia, efetividade, eficiência.

Dez anos após a promulgação da Constituição Federal de 1988, foi expedida a lei 9.956/9833, que introduziu uma série de exigências para o funcionamento e para o encerramento das atividades das operadoras de saúde suplementar. Dois anos após, em 2000, por meio da lei 9.961/004, foi criada a Agência Nacional de Saúde (ANS), autarquia sob regime especial, vinculada ao $\mathrm{Mi}$ nistério da Saúde, como órgão de regulação, normatização, controle e fiscalização das atividades que garantam a assistência suplementar à saúde. Desta forma, tornou-se responsabilidade da ANS assegurar a prestação de serviços aos usuários, introduzir os processos regulatórios para o setor de saúde suplementar e estabelecer regras para as relações entre operadoras de planos de saúde, beneficiários e prestadores de serviços de saúde ${ }^{4,5}$. Um mês antes da sua criação, em dezembro de 1999, existiam aproximadamente 2.639 operadoras de saúde suplementar em atividade que atendiam em torno de 30 milhões de usuários ${ }^{6}$

Em estudo realizado por Salles ${ }^{7}$, foi observado que as operadoras com menos de 10.000 beneficiários, que são a grande maioria das operadoras no mercado de saúde suplementar, ainda têm uma grande dificuldade de absorver a exi- gência dessa regulação. Uma das consequências resultantes desse cenário foi a integração vertical das operadoras de pequeno porte, conforme discutido por Leandro ${ }^{8}$. O autor discute que os custos crescentes dos serviços médicos e hospitalares, o acesso ao financiamento e os desequilíbrios financeiros colaboram para que operadoras com um maior número de beneficiários tenham melhores condições de competir na oferta de planos de saúde, contribuindo, assim, para uma maior concentração no mercado. A análise realizada por Nogueira ${ }^{9}$ levou à conclusão de que as operadoras com menos de 5 mil beneficiários tendem a apresentar elevado grau de insolvência, o que se constatou quando esses dados foram cruzados com o resultado da análise do grupo de operadoras em regimes especiais e em plano de recuperação. Albuquerque et al. ${ }^{10}$ mostram que o mercado de planos de assistência média acompanhou o crescimento da população brasileira, porém a maioria dos beneficiários está concentrada em poucas operadoras.

O Ciclo produtivo do setor de saúde é composto por diversos "players": operadoras de planos de saúde, prestadores, beneficiários e, externamente, o governo, que conduz e regula a política econômica e de saúde. As operadoras de saúde são remuneradas através das contraprestações pagas mensalmente pelos seus beneficiários, que são atendidos pelas redes de prestadores credenciados ou através dos seus serviços próprios.

A relação entre as despesas e a receita das operadoras de saúde suplementar pode ser medida através da sinistralidade. Segundo Pires ${ }^{11}$, a sinistralidade é um índice calculado através da razão entre sinistros realizados (custos de assistência) e o prêmio (receitas da assistência), medida em percentual. O conceito é usado pelas operadoras de saúde como balizador na hora do reajuste de preços. A apuração dos últimos 12 meses da receita versus despesa indica se o contrato é financeiramente compensador para as partes ou se o valor pago na mensalidade é justo e mantém a relação contratual equilibrada, considerando os sinistros ou as despesas geradas com esse contrato ${ }^{12}$.

Pode-se, assim, medir a sinistralidade de um grupo ou de toda a carteira de beneficiários de um determinado plano de saúde. A média tida como aceitável pela maior parte das empresas do ramo é de $75 \%{ }^{11}$. Essa medida permite ao plano de saúde custear suas despesas administrativas e comerciais e ter uma margem de lucro que viabilize o negócio. Com valores acima desse percentual, considera-se que determinada apólice ou determinado contrato é deficitário/ $\mathrm{a}^{13}$. 
Em debates promovidos pela Fundação Getúlio Vargas (FGV), em 2010, foi discutida a obrigatoriedade imposta pela ANS para acatar os novos procedimentos no rol de cobertura e quais as consequências financeiras para as operadoras, sob pena de multa pelo não cumprimento ${ }^{14}$. De acordo com Fernandes et al. ${ }^{15}$, as sanções impostas pela ANS impactam diretamente o balanço financeiro das empresas, afetando diretamente a sobrevida das empresas de menor porte.

Em 2012, a Federação Nacional de Saúde Suplementar (FenaSaúde) alegava que as despesas das operadoras tinham aumentado muito e que seria necessário um ajuste maior para cobrir os custos e sobrar uma margem mais significante e atraente. Segundo Cechin, diretor executivo da FenaSaúde, "A tensão nesta corda está sendo esticada e, frente ao que está acontecendo com as despesas, não há como imaginar que esses planos coletivos não tenham reajustes importantes"16. Essas afirmações feitas em 2012 foram confirmadas a posteriori, quando foi relatado o grande aumento da sinistralidade das operadoras de planos de saúde, que em 2011 era 82,4\% e em 2012 foi $85 \%{ }^{17}$, um aumento considerável de $2,6 \%$. Consequentemente, houve redução nas margens de lucro de tais operadoras, impactando fortemente os resultados financeiros e trazendo riscos a médio e longo prazo. Nogueira ${ }^{9}$ observou que quanto menor o porte da operadora menor é o índice de Sinistralidade. Segundo o autor, isso não significa que essa tendência sinaliza um melhor controle das operadoras de pequeno porte sobre seus custos assistenciais, mas sim uma distorção provocada por erro no lançamento contábil e pela confusão causada entre a compreensão de despesas administrativas e despesas assistenciais.

Ultimamente, dados alarmantes e altamente preocupantes vêm surgindo, por parte das operadoras de saúde, sobre o aumento da sinistralidade e seu potencial risco ${ }^{18,19}$. Contudo, não existe, até o momento, descrição concreta do seu real comportamento temporal. Este é um estudo descritivo, com o objetivo de compreender as mudanças ocorridas no setor de saúde suplementar através da evolução temporal da sinistralidade das operadoras de saúde suplementar no Brasil, no período de 2003 a 2014. Também serão analisadas, neste mesmo período, a taxa de cobertura (que descreverá a demanda pelo serviço) e do número de operadoras em atividade no Brasil (indicando a oferta do serviço).

\section{Metodologia}

Para melhor delimitação do estudo, foram consideradas operadoras de planos de saúde com atuação nacional com dados definidos e coletados pela Agência Nacional de Saúde ${ }^{20}$ (ANS) durante o período de 2003 a 2014, assim como dados macroeconômicos coletados no site do Instituto Brasileiro de Geografia e Estatística ${ }^{21}$ (IBGE). Nesses sites foram coletadas as variáveis: receita total - soma das contraprestações efetivas informadas pelas operadoras à ANS; despesa total soma das despesas informadas pelas operadoras à ANS; número de beneficiários - pessoa física, titular ou dependente, que possui direitos e deveres definidos em legislação e em contrato assinado com a operadora de plano privado de saúde, para garantia da assistência médico-hospitalar; número de operadoras em atividade - pessoa jurídica constituída sob a modalidade empresarial, associação, fundação, cooperativa ou entidade de autogestão, obrigatoriamente registrada na ANS, que opera ou comercializa planos privados de assistência à saúde; e população residente no Brasil - estimativa do tamanho da população brasileira realizada pelo IBGE. Todas as séries históricas podem ser observadas a seguir na Tabela 1.

Tabela 1. Séries históricas do número de beneficiários de planos privados de assistência médica, registro do número de operadoras em atividade de planos privados de assistência médica, receitas e despesas nominais dos planos privados de assistência médica e população residente estimada. (Brasil, 2003-2014).

\begin{tabular}{cccccc}
\hline Ano & $\begin{array}{c}\text { B } \\
\text { (Milhões) }\end{array}$ & $\begin{array}{c}\text { R } \\
\text { (R\$ bilhões) }\end{array}$ & $\begin{array}{c}\text { D } \\
\text { (R\$ bilhões) }\end{array}$ & OA & $\begin{array}{c}\text { PE } \\
\text { (Milhões) }\end{array}$ \\
\hline 2003 & 32,07 & 28,24 & 22,97 & 2273 & 176,87 \\
2004 & 33,84 & 32,03 & 26,05 & 2176 & 181,58 \\
2005 & 35,44 & 36,53 & 29,74 & 2089 & 184,18 \\
2006 & 37,25 & 41,72 & 33,27 & 2065 & 186,77 \\
2007 & 39,32 & 51,11 & 41,20 & 1928 & 184,01 \\
2008 & 41,47 & 59,50 & 47,84 & 1760 & 189,61 \\
2009 & 42,56 & 64,47 & 53,52 & 1693 & 191,51 \\
2010 & 44,92 & 72,92 & 59,17 & 1616 & 190,73 \\
2011 & 46,15 & 82,61 & 68,10 & 1598 & 192,38 \\
2012 & 47,89 & 93,13 & 79,10 & 1535 & 193,98 \\
2013 & 49,55 & 108,22 & 90,62 & 1469 & 201,06 \\
2014 & 50,81 & 124,46 & 105,69 & 1425 & 202,80 \\
\hline
\end{tabular}

Fontes: DIOPS/ANS/MS ${ }^{20}$ e IBGE ${ }^{21}$; (B) Beneficiários, (R) Receitas, (D) Despesas, (OA) Operadoras em Atividade e (PE) População residente estimada. 
A partir dessas variáveis, foram calculadas as seguintes: sinistralidade (S), que representa a razão entre a despesa assistencial e a receita de contraprestações das operadoras, e a taxa de cobertura (TC), dada pela razão entre o número de beneficiários e a população estimada.

As tendências temporais foram verificadas com o uso de Regressão Linear Simples ${ }^{22,23}$. Para tal, foram assumidas, no seguinte modelo de regressão linear $Y=\beta_{0}+\beta_{1} X+\varepsilon$, as variáveis sinistralidade, número de operadoras e taxa de cobertura como variável dependente do modelo $(Y)$, e o tempo, em anos, como variável independente $(X)$, dessa forma, o coeficiente angular do modelo $\left(\beta_{1}\right)$ estimado fornecerá a direção e a magnitude das flutuações. Para $\beta_{1}$ positivo, a série será caracterizada com uma tendência de crescimento. Já o sinal negativo de $\beta_{1}$ indicará uma tendência de decrescimento. Quando $\beta_{1}=0$ tem-se ausência de tendência. A taxa de crescimento das séries foi obtida também através de Regressão Linear Simples, com o ajuste de modelos log-lineares ${ }^{24}$. Utilizamos o software livre R versão $3.2 .3^{25}$ para realizar as análises, e em todos os testes de hipótese realizados adotou-se um nível de significância de $5 \%$.

\section{Resultados}

Observamos, na sequência, na Tabela 2, que a sinistralidade variou entre $79,75 \%$ (observado em 2006) e $84,93 \%$ (observado em 2012), com uma média anual de $82,08 \%$, e desvio padrão de 1,65\%. A Taxa de Cobertura apresentou média de $21,95 \%$ e desvio padrão de $2,39 \%$, em que o valor mínimo $(18,13 \%)$ foi observado no início do período estudado (2003), e o máximo, por seu turno, no final (2014). Ao analisarmos o número de Operadoras em Atividade, verificamos o comportamento inverso ao observado para a taxa de

Tabela 2. Estatística Descritiva das séries de Sinistralidade (\%), Taxa de Cobertura (\%) e Número de Operadoras em Atividade (Brasil, 2003-2014).

\begin{tabular}{lrrrr}
\hline \multicolumn{1}{c}{ Variável } & Média & $\begin{array}{c}\text { Desvio } \\
\text { Padrão }\end{array}$ & Máximo & Mínimo \\
\hline Sinistralidade & 82,08 & 1,65 & 84,93 & 79,75 \\
Taxa de & 21,95 & 2,39 & 25,05 & 18,13 \\
Cobertura & & & & \\
Operadoras & 1802,25 & 280,37 & 2273,00 & 1425,00 \\
\hline
\end{tabular}

Fonte: Autoria própria. cobertura, em que o máximo (2.273 operadoras) ocorreu no início do período estudado (2003), e o mínimo (1.425 operadoras), em 2014.

Os resultados observados na Tabela 3 e na Figura 1 mostram que a sinistralidade apresentou uma leve tendência ao crescimento no período ( $p$ $=0,0044)$, com uma taxa anual de crescimento de $0,44 \%$, confirmando as hipóteses levantadas em trabalhos e discussões anteriores ${ }^{16-19}$. Em média, o crescimento anual da sinistralidade é de $0,36 \%$. A quantidade de operadoras no Brasil apresentou uma tendência de decrescimento no mesmo período $(p=0,0000)$. Em média, 80,09 operadoras saem de operação por ano no país, com uma taxa de decréscimo de $4,43 \%$. Esse resultado pode ser justificado, também, pela dificuldade das operadoras de pequeno porte (com menos de 10.000 beneficiários) em se adequar às exigências de regulação feitas pela $\mathrm{ANS}^{7,9}$, assim como pela integração vertical dessas operadoras de menor porte ${ }^{8}$.

A Taxa de Cobertura dos planos de saúde apresentou uma tendência de crescimento ( $p$ $=0,0000)$ dentro do período. A proporção de brasileiros que são usuários de planos de saúde cresce em média $0,68 \%$ ao ano, sendo que esse valor representa uma taxa de crescimento de $3,16 \%$ ao ano. Esse comportamento de aumento do número de beneficiários já foi discutido por Albuquerque et al. $^{10}$ e mostra que o número de beneficiários aumenta em um ritmo maior do que o crescimento da população.

\section{Discussão}

Os resultados observados levantam sinal alarmante, pois, mesmo com o aumento da demanda, observa-se uma diminuição do número de operadoras em atividade no país. Quais seriam os motivos reais dessa relação inversa de demanda versus oferta, já que, econômica e financeiramente falando, seria um setor altamente atrativo para o investimento do capital privado e abertura de novas empresas? Questiona-se também: esse caminho levará à formação de oligopólios, monopólios e, por fim, ao colapso do sistema de saúde suplementar, que poria em risco também o já deficiente sistema de saúde público (SUS)? Ou levaria a uma seleção exclusiva de operadoras mais preparadas estruturalmente para absorver a demanda com risco de oligopolização e cartelização da área de saúde?

Todos os processos regulatórios introduzidos pelo governo desde a década de 1990 tiveram como objetivo corrigir erros de implantação do 
Tabela 3. Avaliação da tendência das séries de Sinistralidade, Taxa de Cobertura e Número de Operadoras em Atividade (Brasil, 2003-2014).

\begin{tabular}{lrrcc}
\hline \multicolumn{1}{c}{ Variável } & \multicolumn{1}{c}{$\beta_{0}$} & $\beta_{1}$ & P-valor $\left(\beta_{1}\right)$ & Taxa de Crescimento (\% ao Ano) \\
\hline Sinistralidade & $-645,79$ & 0,36 & 0,0044 & 0,44 \\
Taxa de Cobertura & $-1350,52$ & 0,68 & 0,0000 & 3,16 \\
Operadoras & 162671,86 & $-80,09$ & 0,0000 & $-4,43$ \\
\hline
\end{tabular}

Fonte: Autoria própria.

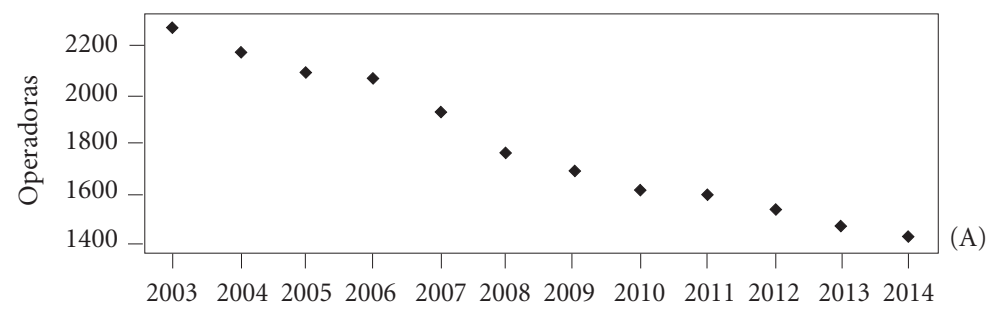

Anos

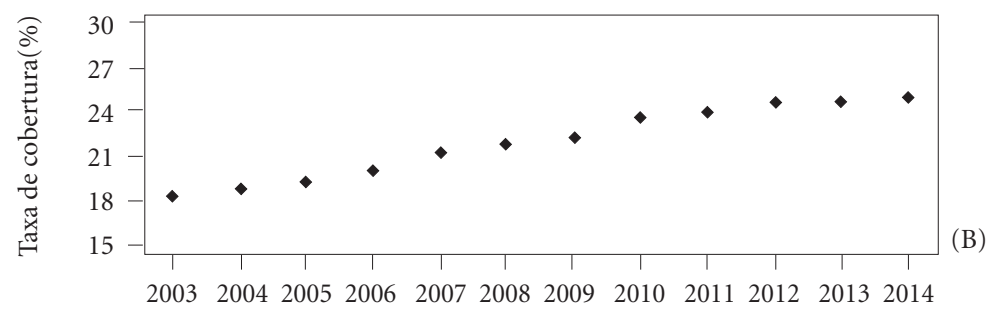

Anos

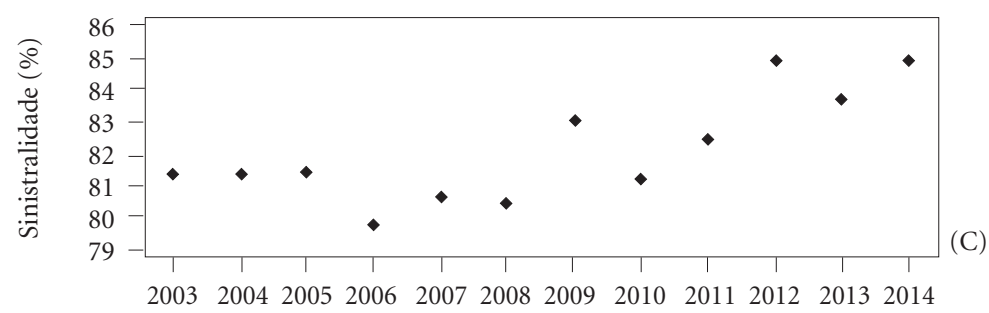

Anos

Figura 1. Séries históricas do número de operadoras de planos de saúde em atividade (A), taxa de cobertura de pessoas que utilizam planos de saúde suplementar (B) e taxa de sinistralidade das operadoras de planos privados de saúde ᄀ. Brasil, 2003-2014.

passado e filtrar e readequar as operadoras de planos de saúde suplementar que, sabendo dos riscos de negócios, deveriam focar na atenção à saúde e na satisfação dos beneficiários, mantendo assim uma estrutura econômica e de operação para que pudessem honrar com seus compromis$\operatorname{sos}^{1,2}$. Encontra-se aí mais uma justificativa para a diminuição da quantidade de operadoras de planos de saúde suplementar no Brasil, demonstrando que existia um número muito grande de operadoras funcionando de forma inadequada, pondo em risco a relação com os beneficiários. Atualmente, crescem as queixas de má prestação de serviços e aumenta o número de operadoras com planos suspensos por não cumprimento das normas regulatórias ${ }^{26,27}$. Outros desdobra- 
mentos sucederam paralelamente às transformações legais ocorridas, criando um ciclo vicioso e contribuindo para a diminuição da quantidade de operadoras, como: aumento da demanda por serviço, criação de novas tecnologias, medicalização, formação flexneriana, mudanças de regras operacionais $^{7,9,13}$ e intervencionismo excessivo judiciário/estatal.

$\mathrm{O}$ aumento da sinistralidade põe em risco a grande malha assistencial de saúde suplementar do Brasil, e o esclarecimento do comportamento e sua tendência permitirão, por conseguinte, traçar planejamento adequado para enfrentar tal risco. A diminuição do número de operadoras poderá acender uma luz amarela, já que é um setor deficiente e não cobre toda a demanda, alertando a respeito da sobrevida no curto e médio prazo para a saúde suplementar. Em 2007, Ocké-Reis ${ }^{28}$ já havia observado uma maior concentração do mercado de planos de saúde, sinalizando uma tendência ao maior refino na seleção das operadoras atuantes, oligopolizando o mercado e deixando apenas as operadoras melhor estruturadas.

Esse cenário se apresenta como um fator impactante para a atração de novas operadoras de planos de saúde suplementar e manutenção das já existentes, pois diminui as margens de lucro, não tornando o investimento atrativo. Por outro lado, a sinistralidade vem sendo controlada rigidamente por ações administrativas das operadoras de planos de saúde que, no entanto, são contrárias às normas regulatórias do governo e que contrariam também o livre acesso ao beneficiário. Desse modo, podem estar causando um efeito revés com aumento da judicialização ${ }^{29,30}$ e sansões administrativas da ANS para as operadoras, com consequente diminuição do seu número.
Com base nos dados coletados pela $\mathrm{ANS}^{6}$, existe um alerta ainda maior: o envelhecimento populacional ${ }^{26,31-33}$ e o aumento de doenças crônicas $^{34}$. Segundo o IBGE $^{35}$, até 2060 o número de idosos no Brasil quadruplicará; as estimativas apontam que o país terá o mesmo número de habitantes que em 2025 (218,2 milhões), só que $26,7 \%$, ou seja, 58,4 milhões de pessoas serão idosas. Diante de tudo isso, somos incitados a refletir sobre soluções urgentes para todo o sistema de saúde do Brasil para que não corra o risco de um dia entrar em colapso.

\section{Conclusão}

Conclui-se que a tendência de crescimento da sinistralidade, observada entre os anos de 2003 e 2014, oferece riscos à sobrevida e à abertura de novas operadoras. Mesmo com um índice elevado de sinistralidade, a saúde suplementar vem se adequando ao longo dos anos, pondo em risco a relação com os beneficiários e prestadores, sendo fortemente vigiada pela ANS. Por outro lado, a diminuição do número de operadoras pode estar conduzindo o país a uma oligopolização do setor, com uma demanda crescente pelo serviço, representado pelo crescimento da taxa de cobertura.

Essa diminuição está também associada aos processos regulatórios, que, via de regra, normatizam a relação do setor com o beneficiário, que, do ponto de vista da relação, é o elemento mais frágil. Na verdade, o que chama a atenção é que o número de operadoras está reduzindo, enquanto as dificuldades operacionais estão aumentando, e a margem de lucro, diminuindo. 


\section{Colaboradores}

AAS Araújo foi responsável pela concepção do projeto, desenvolvimento da pesquisa e redação do artigo. JRS Silva trabalhou no desenvolvimento da pesquisa, análise estatística e na redação final do trabalho.

\section{Referências}

1. Escrivão Junior A, Koyama MF. O relacionamento entre hospitais e operadoras de planos de saúde no âmbito do Programa de Qualificação da Saúde Suplementar da ANS. Cien Saude Colet 2007; 12(4):903-914.

2. Bahia L. Planos privados de saúde: luzes e sombras no debate setorial dos anos 90. Cien Saude Colet 2001; 6(2):329-339.

3. Brasil. Lei $\mathrm{n}^{\circ}$ 9.956, de 03 de Junho de 1998. Dispõe sobre mecanismos de regulação nos Planos e Seguros Privados de Assistência à Saúde. Diário Oficial da União 1998; 4 jun.

4. Brasil. Lei № 9.961, de 28 de janeiro de 2000. Cria a Agência Nacional de Saúde Suplementar - ANS e dá outras providências. Diário Oficial da União 2000; 29 jan.

5. Costa $\mathrm{N}$ do R. O regime regulatório e o mercado de planos de saúde no Brasil. Cien Saude Colet 2008; 13(5):1453-1462.

6. Agência Nacional de Saúde (ANS). Caderno de informações de saúde suplementar: Beneficiário, operadoras e planos. Rio de Janeiro: ANS; 2014.

7. Salles OR. Adaptação das Operadoras de Planos de Saúde de Pequeno Porte ao Plano de Contas Padrão da ANS [dissertação]. Rio de Janeiro: Escola Nacional de Saúde Pública; 2004.

8. Leandro T. Defesa da Concorrência e Saúde Suplementar: A Integração Vertical entre Planos de Saúde e Hospitais e seus efeitos no Mercado [dissertação]. Brasília: Universidade de Brasília; 2010.

9. Nogueira CAL. Análise da estrutura econômica do mercado de saúde suplementar: solvência do mercado e benefícios da concentração. Rio de Janeiro: Fundação Oswaldo Cruz; 2004.

10. Albuquerque C, Piovesan MF, Santos IS, Martins ACM, Fonseca AL, Sasson D, Simões KA. A situação atual do mercado da saúde suplementar no Brasil e apontamentos para o futuro. Cien Saude Colet 2008; 13(5):14211430.

11. Pires FMS. Estudo do impacto da medicina preventiva na diminuição da sinistralidade dos planos de saúde e sua aplicação ao sistema SAMMED/FUSEX. Rio de Janeiro: Escola de Saúde do Exército; 2008.

12. Silva RV. Sinistralidade e relação contratual. J Valor Econômico [Internet]. 2014 [cited 2014 Dec 2]; Available from: http://alfonsin.com.br/sinistralidade-e-relao-contratual/

13. Fernandes F. A Sinistralidade Crescente no Sistema de Saúde [Internet]. 2006 [cited 2012 Oct 11]; Available from: http://www.athongroup.com.br/materias_publicadas/pdf/2006_11_06_sinistralidade.pdf

14. Balzan MV, Ceschin M, Akl M. O impacto do novo rol de procedimentos da ANS nos custos das operadoras de planos de saúde [Internet]. Fundação Getúlio Vargas. 2010 [cited 2014 Dec 2]; Available from: http:// gvsaude.fgv.br/pt-br/ans

15. Fernandes F, Ferreira ME, Rodrigues ER. Análise de Rentabilidade Utilizando o Modelo Dupont: Estudo de Caso em uma Operadora de Planos de Saúde. Rev. Gestão em Sist. Saúde 2014;3(2):30-44. 
16. Fundação Apoio ao Desenvolv. Científico e Tecnológico do Hosp. da Univ. Brasília (FAHUB). Operadoras de planos de saúde falam em reajuste maior em 2013 [Internet]. 2012. [cited 2014 Dec 2]. Available from: http:// www.fahub.org.br/index.php/linha-direta/164-operadoras-de-planos-de-saude-falam-em-reajuste-maiorem-2013

17. Koike B. Com despesa em alta, hospitais preveem crescer pouco. J Valor Econômico [Internet]. 2013 [cited 2014 Dec 2]; Available from: http://www.valor.com. br/empresas/3144764/com-despesa-em-alta-hospitais-preveem-crescer-pouco

18. Guimarães ALS, Nossa V. Working Capital, Profitability, Liquidity and Solvency of Healthcare Insurance Companies. BBR - Brazilian Bus. Rev. 2010; 7(2):37-59.

19. Kudlawicz C, Santos JL. Perfil financeiro das empresas brasileiras operadoras de planos de saúde: um estudo exploratório. Conhecimento Interativo [Internet]. 2014 Jun 30 [cited 2015 Jun 27]; 7(1):30-47. Available from: http://app.fiepr.org.br/revistacientifica/index.php/ conhecimentointerativo/article/view/135

20. Agência Nacional de Saúde Suplementar (ANS). Caderno de Informação da Saúde Suplementar: beneficiários, operadoras e planos [Internet]. Rio de Janeiro: Ministério da Saúde (MS); 2015. [cited 2015 Jun 27]. Available from: http://www.ans.gov.br/portal/site/informacoesss/informacoesss.asp

21. Instituto Brasileiro de Geografia e Estatística (IBGE). Projeção da população do Brasil e das Unidades da Federação [Internet]. População. 2016. [cited 2016 Feb 8]. Available from: http://www.ibge.gov.br/apps/populacao/projecao/

22. Pearson K, Yule GU. The Law of Ancestral Heredity. Biometrika 1903; 2(2):211-228.

23. Yule GU. On the Theory of Correlation. J R. Stat. Soc. 1897; 60(4):812-854.

24. Gujarati DN. Econometria Básica. $3^{\text {rd }}$ ed. São Paulo: Pearson Makron Books; 2005.

25. The R Core Team. R: A Language and Environment for Statistical Computing [Internet]. Vienna: R Foundation for Statistical Computing; 2016. [cited 2015 Jun 27]. Available from: https://www.r-project.org/

26. Vieira Júnior WM, Martins M. The elderly and healthcare plans in Brazil: analysis of the complaints received by the National Regulatory Agency for Private Health Insurance and Plans. Cien Saude Colet 2015; 20(12):3817-3826.

27. Machado JRO. Negativas de cobertura pelas operadoras de planos de saúde: análise das denúncias de beneficiários encaminhadas à Agência Nacional de Saúde Suplementar (ANS). Rio de Janeiro: Fundação Osvaldo Cruz; 2011.
28. Ocké-Reis CO. Os desafios da ANS frente à concentração dos planos de saúde. Cien Saude Colet 2007; 12(4):1041-1050.

29. Gonçalves T, Machado FJ. Judicialização da saúde suplementar. Anuário da Produção Iniciação Científica Discente 14(24):43-55.

30. Mandarino RP, D Arbo Alves MH. Ativismo judicial e judicialização da política da relação de consumo: uma análise do controle jurisdicional dos contratos de planos de saúde privado no estado de São Paulo. Rev. Bras. Polit. Públicas 2015; 5(Edição Especial-Ativismo Judicial).

31. Stivali M. Regulação da saúde suplementar e estrutura etária dos beneficiários. Cien Saude Colet 2011; 16(9):3729-3739.

32. Sá MC, Almeida MR, Costa JAF, Abreu EAB, Viana AF. Os riscos do Mercado de Saúde Suplementar: Uma revisão bibliográfica do impacto da transição demográfica e epidemiológica nas operadoras de plano de saúde no Brasil. In: X Simpósio de Excelência em Gestão e Tecnologia. Resende - RJ: 2013.

33. Nunes A. O envelhecimento populacional e as despesas do Sistema Único de Saúde. In: Camarano AA, editor. Os novos idosos brasileiros. Muito além dos 60? Rio de Janeiro: Instituto de Pesquisa Econômica Aplicada; 2004. p. 427-450.

34. Barros MBA, César CLG, Carandina L, Torre GD. Desigualdades sociais na prevalência de doenças crônicas no Brasil, PNAD-2003. Cien Saude Colet [Internet]. 2006 Dec [cited 2016 Jul 19]; 11(4):911-926. Available from: http://www.scielo.br/scielo.php?script=sci_arttext\&pid=S1413-81232006000400014\&lng=pt\&n$\mathrm{rm}=\mathrm{iso} \& \mathrm{t} \operatorname{lng}=\mathrm{pt}$

35. Instituto Brasileiro de Geografia e Estatística (IBGE). Censo Demográfico 2010. Rio de Janeiro: IBGE; 2010.

Artigo apresentado em 16/02/2016

Aprovado em 13/09/2016

Versão final apresentada em 15/09/2016 